\title{
Hermansky-Pudlak syndrome with neutropenia
}

INSERM

\section{Source}

INSERM. (1999). Orphanet: an online rare disease and orphan drug data base.

Hermansky-Pudlak syndrome with neutropenia. ORPHA:183678

Hermansky-Pudlak syndrome type 2 (HPS-2) is a type of Hermansky-Pudlak syndrome (HPS; see this term), a multi-system disorder characterized by oculocutaneous albinism, bleeding diathesis and neutropenia. 